\title{
The context counts: Congruent learning and testing environments prevent memory retrieval impairment following stress
}

\author{
Lars Schwabe ANd Oliver T. Wolf \\ Ruhr University Bochum, Bochum, Germany
}

\begin{abstract}
Stress before retention testing impairs memory, whereas memory performance is enhanced when the learning context is reinstated at retrieval. In the present study, we examined whether the negative impact of stress before memory retrieval can be attenuated when memory is tested in the same environmental context as that in which learning took place. Subjects learned a 2-D object location task in a room scented with vanilla. Twenty-four hours later, they were exposed to stress or a control condition before memory for the object location task was assessed in a cued-recall test, either in the learning context or in a different context (unfamiliar room without the odor). Stress impaired memory when assessed in the unfamiliar context, but not when assessed in the learning context. These results suggest that the detrimental effects of stress on memory retrieval can be abolished when a distinct learning context is reinstated at test.
\end{abstract}

Our ability to remember past experiences is subject to many influences. One of these influences is stress, which triggers a multitude of mental, behavioral, and physiological responses whose aim is to reinstate homeostasis. The nature of the stress effects on memory depends crucially on the timing of the stress exposure (Joels, $\mathrm{Pu}$, Wiegert, Oitzl, \& Krugers, 2006). Both facilitating and detrimental effects can be observed when individuals are stressed before learning (Kirschbaum, Wolf, May, Wippich, \& Hellhammer, 1996; Payne et al., 2006; Schwabe, Bohringer, Chatterjee, \& Schachinger, 2008). Stress immediately after learning enhances subsequent memory, which is most likely due to the beneficial effects of stress on memory consolidation (Cahill, Gorski, \& Le, 2003; Roozendaal, Okuda, Van der Zee, \& McGaugh, 2006; Smeets, Otgaar, Candel, \& Wolf, 2008). In contrast, stress before retention testing has mainly impairing effects on memory retrieval (Buchanan, Tranel, \& Adolphs, 2006; de Quervain, Roozendaal, Nitsch, McGaugh, \& Hock, 2000; Kuhlmann, Piel, \& Wolf, 2005). Recent studies have suggested that these effects of stress on memory are due to the action of stress hormones and neurotransmitters, such as cortisol and noradrenaline, on the amygdala, the hippocampus, and adjacent medial temporal lobe structures (de Quervain et al., 2003; Oei et al., 2007; for reviews, see Diamond, Campbell, Park, Halonen, \& Zoladz, 2007; Lupien \& Lepage, 2001; McGaugh \& Roozendaal, 2002; Wolf, 2008). How memory impairments after stress can be prevented is an important issue in current research on stress and memory. Converging evidence from animal and human studies indicates that $\beta$-adrenergic antagonists such as propranolol abolish the effects of stress (hormones) on memory retrieval (de Quervain, Aerni, \& Roozendaal, 2007; Roozendaal, de Quervain, Schelling, \& McGaugh, 2004; Schwabe, Römer, et al., 2009). However, the potential side effects of beta-blockers argue against their comprehensive use, for example, in educational settings. Other, less harmful attenuators of stress effects on memory retrieval would be highly desirable.

Besides stress, another variable that influences our memory is the context in which retention testing takes place. Typically, individuals show better memory when the learning environment is reinstated at test than when testing occurs in a different environmental context (for reviews, see Smith \& Vela, 2001; Tulving \& Thompson, 1973). In a classic example of context-dependent memory, scuba divers learned word lists on land or underwater. Their memory for these words was tested subsequently in either the same or the opposite context. It was found that those divers who had to recall the words in the original environment remembered significantly more words than did those requested to change environments (Godden \& Baddeley, 1975). The beneficial effect of congruent learning and retrieval environments can be explained by assuming that incidental background (i.e., contextual) stimuli are stored in memory and that such stimuli can cue memory for material learned contiguously with the incidental context (Glenberg, 1997; Smith \& Vela, 2001). Context effects on memory can be implicit or explicit (Chun \& Jiang, 2003; Cohen \& Eichenbaum, 1993). At a neural level, contextdependent memory is mediated by the prefrontal cortex and the medial temporal lobe (Chun \& Phelps, 1999; Rasch, Büchel, Gais, \& Born, 2007; Wagner, Desmond, Glover, \& Gabrieli, 1998). Interestingly, these brain areas 


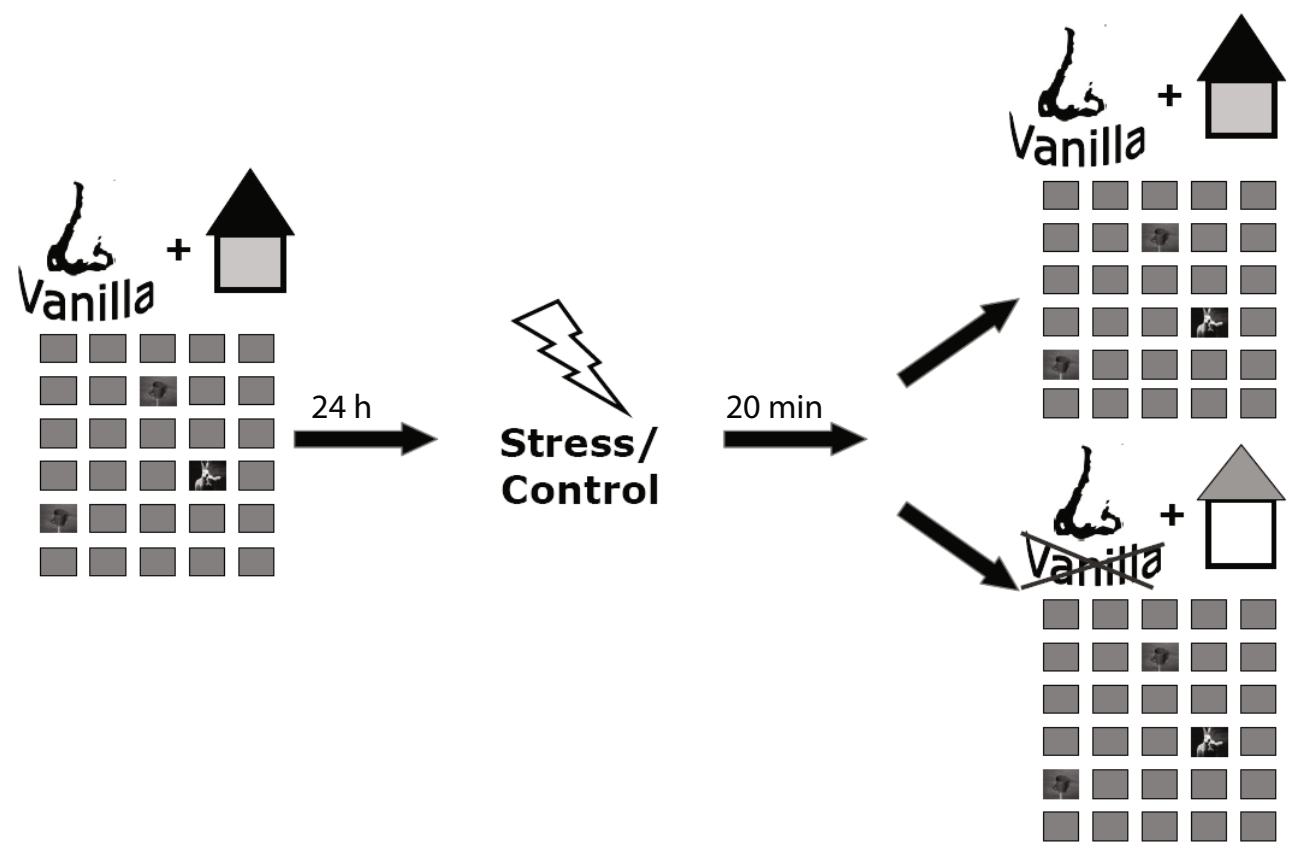

\begin{abstract}
Figure 1. Overview of the study procedure. Subjects were exposed to stress (socially evaluated cold pressor test) or a control condition before their memory for an object location task that they had been trained on the day before was tested. Importantly, during learning, we presented a vanilla odor. Memory testing took place either in the same room where learning had taken place and in the presence of the vanilla odor (i.e., in a congruent context) or in a different room without the vanilla odor (i.e., in an incongruent context). The nose and the house represent the odor and the spatial environment, respectively, in the drawing.
\end{abstract}

contain a high density of glucocorticoid and mineralocorticoid receptors, the two receptor types that mediate cortisol actions, which suggests a high sensitivity to stress (de Kloet, Vreugdenhil, Oitzl, \& Joels, 1998).

Despite this, only very little is known about the possible interplay between stress and contextual effects on memory. Very recently, we investigated the impact of stress on the formation of context-dependent memories (Schwabe, Bohringer, \& Wolf, 2009). Subjects were exposed to stress or a control condition before they learned an object location task that was presented in a room scented with vanilla. Memory for this task was tested on the following day, either in the same room as that in which learning had taken place, again scented with vanilla, or in a different room without the odor. What we found was that stress before learning disrupted the context-dependent memory enhancement observed in nonstressed control subjects, suggesting that stress impaired the integration of contextual cues in the memory trace. Once contextually enriched memories are created, they are associated with superior performance when tested in the appropriate context (Smith \& Vela, 2001). Whether context-dependent memories are still susceptible to the influence of stress after they have been established is not known. Might matching learning and retrieval contexts even prevent the detrimental impact of stress on memory retrieval?

In the present experiment, we tested the hypothesis that the memory-impairing effect of stress administered before retrieval is attenuated when learning and testing contexts match. As in our previous study (Schwabe, Bohringer, \& Wolf, 2009), subjects were trained in a 2-D object location task. We presented a vanilla odor during learning, since earlier studies had shown that odors are very potent context cues facilitating memory (Rasch et al., 2007). Twenty-four hours after learning, the subjects were exposed to either a stress protocol (socially evaluated cold pressor test [SECPT]; Schwabe, Haddad, \& Schachinger, 2008) or a control condition before their memory for the object location task was tested. Importantly, retention testing took place in a context either congruent (same room, vanilla odor present) or incongruent (different room, no vanilla odor) with the learning context. Please note that we did not aim to test the effects of the different context components (odor, room) but to create a distinct context that might support memory.

\section{METHOD}

\section{Subjects}

Seventy-two students at the Ruhr University Bochum (36 men, 36 women; age $[M \pm S E M] ; 25.1 \pm 0.5$ years; body mass index $[M \pm$ $\left.S E M] ; 22.9 \pm 0.3 \mathrm{~kg} / \mathrm{m}^{2}\right)$ participated in the experiment. Participation was restricted to healthy nonsmokers. Women taking oral contraceptives were excluded from participation because oral contraceptives change the neuroendocrine stress response (Kirschbaum, Kudielka, Gaab, Schommer, \& Hellhammer, 1999). The subjects had to refrain from meals, caffeine, alcohol, and taking exercise within the $2 \mathrm{~h}$ prior to the experiment. All the subjects provided written informed consent for their participation in the protocol as approved by the ethics committee of the German Psychological Association. 
Table 1

Subjective Assessments (on a Scale From 0 [Not at All] to 100 [Very Much]) and Blood Pressure Responses (in mmHg) As a Function of Treatment (Control vs. Stress)

\begin{tabular}{llllll}
\hline & \multicolumn{2}{c}{ Control } & & \multicolumn{2}{c}{ Stress } \\
\cline { 2 - 3 } \cline { 5 - 6 } & $M$ & SEM & & $M$ & SEM \\
\hline Subjective Assessments & & & & \\
$\quad$ Painfulness & 2.7 & 2.2 & & $\mathbf{6 4 . 3}$ & $\mathbf{4 . 5}$ \\
$\quad$ Stressfulness & 4.1 & 2.1 & & $\mathbf{4 4 . 6}$ & $\mathbf{5 . 0}$ \\
Unpleasantness & 5.4 & 1.3 & & $\mathbf{6 0 . 9}$ & $\mathbf{5 . 1}$ \\
Systolic Blood Pressure & & & & \\
$\quad$ Before treatment & 116.8 & 2.6 & & 118.6 & 2.0 \\
$\quad$ During treatment & 111.7 & 2.4 & & $\mathbf{1 3 5 . 5}$ & $\mathbf{2 . 5}$ \\
After treatment & 111.2 & 2.5 & & 117.1 & 2.1 \\
Diastolic Blood Pressure & & & & \\
Before treatment & 65.6 & 1.3 & & 68.6 & 1.2 \\
$\quad$ During treatment & 64.6 & 1.4 & $\mathbf{7 9 . 1}$ & $\mathbf{1 . 8}$ \\
After treatment & 65.2 & 1.4 & 67.6 & 1.3 \\
\hline
\end{tabular}

Note-Boldface, significant group differences $(p<.05)$.

\section{Stress Protocol}

The subjects in the stress condition were exposed to the SECPT, as described in detail elsewhere (Schwabe, Haddad, \& Schachinger, 2008). Briefly, they immersed their hand up to and including the wrist for up to $3 \mathrm{~min}$ (or until they could no longer tolerate it) in ice water $\left(0^{\circ}-2^{\circ} \mathrm{C}\right)$. They were monitored by an unfamiliar person and videotaped during hand immersion, since social evaluation is critical for stress induction (Dickerson \& Kemeny, 2004). The subjects in the control condition submerged their hand up to and including the wrist for $3 \mathrm{~min}$ in warm water $\left(35^{\circ}-37^{\circ} \mathrm{C}\right)$; they were neither monitored nor videotaped.

To assess the efficacy of the SECPT, immediately after the SECPT or the control condition, the subjects rated how stressful, painful, and unpleasant they had experienced the treatment to be, on a scale from 0 (not at all) to 100 (very much). In addition, blood pressure measurements were taken immediately before, during, and immediately after the SECPT or the control condition. Moreover, saliva samples were collected by means of Salivette (Sarstedt, Germany) collection devices immediately before, as well as 1, 20, and $30 \mathrm{~min}$ after cessation of, the SECPT or control condition. Free concentrations of cortisol, the stress hormone that is released from the adrenal cortex, were measured from saliva, using an immunoassay (IBL, Hamburg).

\section{Memory Task}

The subjects were presented a computer version of the wellknown card game "Concentration" (also known as "Memory"; created with the biopsychology toolbox developed by Rose, Otto, \& Dittrich, 2008). They saw a configuration of 15 card pairs (5 pictures $\times 6$ rows) showing colored pictures. First, all the cards were shown as gray squares ("laid face down"). Next, the subjects could choose two cards and turn them face up. If the two cards showed the same picture, the subjects could turn the next two cards. If they were not the same, the second card was turned face down again, and the subjects had to continue their search for the matching card. The subjects were instructed to turn over all pairs of matching picture cards as fast as possible. Pictures were taken from the International Affective Picture System (Lang, Bradley, \& Cuthbert, 1997) and varied in emotional valence from neutral to positive and negative (five pictures per emotion category), because previous studies had suggested that stress effects on memory differ for neutral and emotional material (Kuhlmann et al., 2005; Schwabe, Römer, et al., 2009). Negative pictures (arousal ratings taken from Lang et al., 1997: $M \pm$ $S E M, 6.8 \pm 0.3$ ) were associated with significantly higher emotional arousal than were positive pictures $(4.5 \pm 0.1)$, which were more arousing than neutral pictures $(2.9 \pm 0.1 ; p \mathrm{~s}<.001)$. The subjects completed four trials of the memory task during learning on Experimental Day 1. They were asked to memorize the picture locations, since these would be tested later. On the following day, one retention test trial was given. The spatial arrangement of the cards was randomized across subjects but was constant for each subject.

Memory performance was expressed as (1) the time needed to complete the memory game on Day 2 minus the time needed to complete the memory game on the last learning trial $24 \mathrm{~h}$ before and (2) the number of correct card locations (i.e., correct card pair locations without error) on the test trial on Day 2, relative to the number of correct card locations on the last learning trial on the previous day.

\section{Procedure}

The study procedure is summarized in Figure 1. All testing took place between 1:30 and 5:30 p.m. in order to control for the diurnal rhythm of cortisol, which is characterized by a morning peak and an evening nadir (Kirschbaum \& Hellhammer, 1994). On the first experimental day, the subjects performed four trials of the memory task. Importantly, during learning, we presented a vanilla odor.

Twenty-four hours later, the subjects returned to the laboratory and were randomly assigned to one of four experimental groups ( 9 men and 9 women per group): control-condition/congruent-context, stresscondition/congruent-context, control-condition/incongruent-context, and stress-condition/incongruent-context. A baseline measurement of blood pressure was taken, and a first saliva sample was collected. Next, the subjects were exposed either to the control condition or to the SECPT; blood pressure was measured during both manipulations. Immediately thereafter, the subjects rated the previous manipulation with respect to its painfulness, stressfulness, and unpleasantness and gave another saliva sample, and a further blood pressure measurement was taken. After a 15-min break in which the subjects were allowed to read, another saliva sample was collected. Twenty minutes after the cessation of the SECPT/control manipulation, the subjects moved to another room and completed one retention test trial of the memory task. This interval between the stress/control manipulation and the memory test was chosen because previous studies had shown that cortisol concentrations reach peak levels 20-30 min after the SECPT (Schwabe, Bohringer, et al., 2008; Schwabe, Haddad, \& Schachinger, 2008). Importantly, the retention test was given either in the room where learning had taken place the day before and again with the vanilla odor present (congruent context) or in a different room without

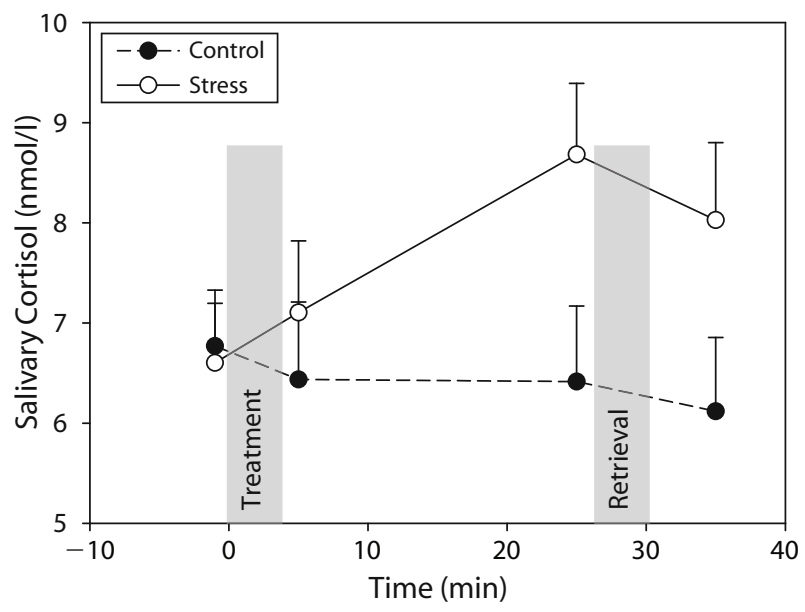

Figure 2. Salivary cortisol concentrations (in nanomoles per liter) in the stress and control groups immediately before as well as 1,20, and $30 \mathrm{~min}$ after the treatment (SECPT vs. control condition). Subjects in the stress group showed a significant increase in salivary cortisol, whereas no such increase was found in controls. Data represent means + standard errors of the means. 
Table 2

Performance in the Control and Stress Groups Expressed As Processing Time and Correct Card Pair Locations Across the Four Training Trials

\begin{tabular}{|c|c|c|c|c|c|c|c|c|c|c|c|c|c|c|c|c|}
\hline \multirow[b]{3}{*}{ Group } & \multicolumn{8}{|c|}{ Processing Time (in Seconds) } & \multicolumn{8}{|c|}{ Correct Card Pair Locations } \\
\hline & \multicolumn{2}{|c|}{1} & \multicolumn{2}{|c|}{2} & \multicolumn{2}{|c|}{3} & \multicolumn{2}{|c|}{4} & \multicolumn{2}{|c|}{1} & \multicolumn{2}{|c|}{2} & \multicolumn{2}{|c|}{3} & \multicolumn{2}{|c|}{4} \\
\hline & $M$ & $\overline{S E M}$ & $M$ & SEM & $M$ & $\overline{S E M}$ & $M$ & SEM & $M$ & $\overline{S E M}$ & $M$ & SEM & $M$ & $\overline{S E M}$ & $M$ & $S E M$ \\
\hline Control & 138.4 & 8.3 & 83.9 & 5.2 & 58.8 & 3.1 & 47.6 & 2.6 & 6.8 & 0.4 & 7.8 & 0.5 & 9.4 & 0.5 & 10.5 & 0.5 \\
\hline Stress & 128.9 & 7.7 & 81.7 & 4.3 & 60.0 & 3.0 & 48.3 & 3.1 & 6.8 & 0.3 & 8.1 & 0.4 & 9.4 & 0.5 & 10.7 & 0.4 \\
\hline
\end{tabular}

the odor (incongruent context). Finally, another saliva sample was collected $30 \mathrm{~min}$ after the end of the stress/control condition.

\section{Statistical Analyses}

Separate ANOVAs with between-subjects factors of treatment (stress vs. control), sex (men vs. women), and context (congruent vs. incongruent context) were conducted on the ratings of painfulness, stressfulness, and unpleasantness. Similarly, changes in cortisol as in systolic and diastolic blood pressure were analyzed by means of separate mixed-design ANOVAs with the between-subjects factors treatment (stress vs. control), sex (men vs. women), and context (congruent vs. incongruent context) and the within-subjects factor time point of measurement. Cortisol values of 6 subjects in the control group ( 3 in the congruent context group) and 4 subjects in the stress group ( 1 in the congruent context group) were missing because these subjects did not provide enough saliva for the biochemical analysis. Memory performance, expressed as difference in processing time and percentage of correct card locations, was assessed by mixed-design ANOVAs with emotional valence (neutral vs. positive vs. negative pictures) as a within-subjects factor and treatment (stress vs. control), context (congruent vs. incongruent context), and sex (men vs. women) as between-subjects factors. All reported $p$ values are two-tailed. Partial eta squared $\left(\eta^{2}\right)$ and Cohen's $d$ were used as a measures of effect size.

\section{RESULTS}

\section{Subjective and Physiological Responses to Stress}

All but 2 of the subjects in the stress group (1 man, 1 woman) submerged their hand in the ice water for the full $3 \mathrm{~min}$. These 2 subjects did not differ in their subjective and physiological stress responses from the rest of the stress group.

Subjective assessments. As was expected and as is indicated in Table 1, the subjects in the stress group experienced the treatment as significantly more painful, stressful, and unpleasant than did controls [all $F_{\mathrm{s}}(1,64)>50$, all $p \mathrm{~s}<.001$, all $\eta^{2} \mathrm{~s}>$.45]. Men and women, as well as the subjects in the congruent and incongruent context conditions, did not differ in their subjective assessments [all $F \mathrm{~s}(1,64)<1.3$, all $p \mathrm{~s}>.25$, all $\eta^{2} \mathrm{~s}<.02$ ].

Systolic and diastolic blood pressure. Exposure to the SECPT led to a significant increase in systolic and diastolic blood pressure [both $F_{\mathrm{s}}(1,64)>15$, both $p \mathrm{~s}<$ .001 , both $\left.\eta^{2} \mathrm{~s}>.19\right]$. As Table 1 shows, the subjects in the stress and control conditions differed during hand immersion, whereas their systolic and diastolic blood pressure was comparable before and after hand immersion in ice or warm water [main effects of time point of measurement, both $F \mathrm{~s}(2,128)>30$, both $p \mathrm{~s}<.001$, both $\eta^{2} \mathrm{~s}>$ .30 ; treatment $\times$ time point of measurement interaction effects, both $F_{\mathrm{s}}(2,128)>40$, both $p \mathrm{~s}<.001$, both $\eta^{2} \mathrm{~s}>$ .40]. Overall, men had higher systolic and diastolic blood pressure than did women [both $F_{\mathrm{s}}(1,64)>8$, both $p \mathrm{~s}<$ .01 , both $\eta^{2} \mathrm{~s}>.12$ ]; however, there was no sex difference in the blood pressure response to the SECPT [both $F_{\mathrm{s}}(1,128)<1.3$, both $p \mathrm{~s}>.25$, both $\left.\eta^{2} \mathrm{~s}<.02\right]$. The subjects in the congruent and incongruent context conditions did not differ in their blood pressure responses [both $F_{\mathrm{s}}(1,64)<2.1$, both $p \mathrm{~s}>.15$, both $\eta^{2} \mathrm{~s}<.03$ ], nor did any further interaction reach significance.

Cortisol. The SECPT caused an increase in salivary cortisol, which was not observed after the control manipulation [treatment $\times$ time point of measurement interaction, $\left.F(3,159)=3.44, p=.018, \eta^{2}=.06\right]$. Figure 2 shows that stressed and control subjects differed, in particular, $20 \mathrm{~min}$ after the SECPT, when retention testing started $(p<.05)$. As in previous studies using the SECPT (Schwabe, Bohringer, et al., 2008; Schwabe, Bohringer, \& Wolf, 2009), men and women did not differ in their cortisol levels, nor was there a difference between the congruent and incongruent context groups (all $F \mathrm{~s}<2.3$, all $p \mathrm{~s}>.15$, all $\left.\eta^{2} \mathrm{~s}<.04\right)$.

\section{Learning Performance}

The subjects showed a significant improvement during learning that was reflected in an increasing number of correct card locations $\left[F(3,192)=67.3, p<.001, \eta^{2}=.49\right]$ and a decreasing processing time $[F(3,192)=180.6, p<$

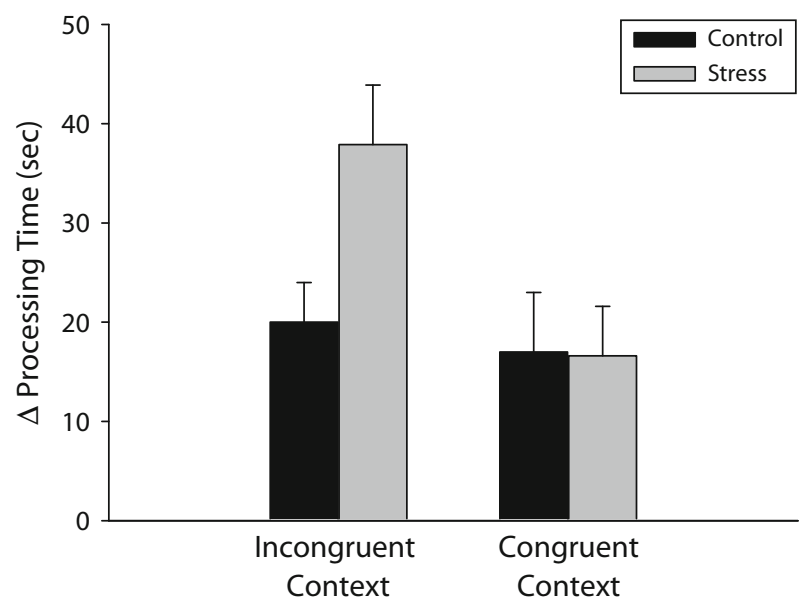

Figure 3. Difference between the times needed to complete the object location task on the test trial and the last learning trial $24 \mathrm{~h}$ before ( $\Delta$ processing time) as a function of treatment (stress vs. control) and context condition (congruent vs. incongruent context). Smaller differences indicate better memory performance. Stress impaired performance in the incongruent context condition, but not in the congruent context condition. Data represent means + standard errors of the means. 


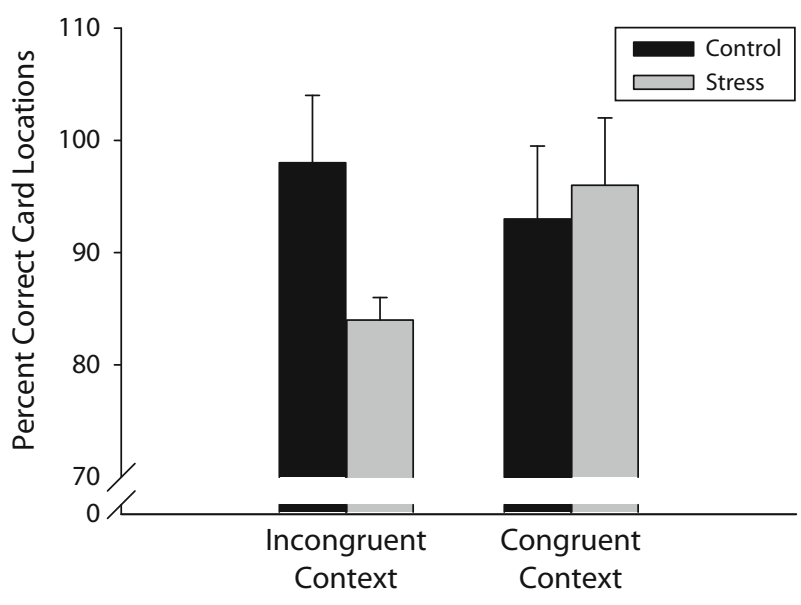

Figure 4. Percentages of correct card locations on the retention test trial, relative to the last learning trial $24 \mathrm{~h}$ before, as a function of treatment (stress vs. control) and context condition (congruent vs. incongruent context). Stress reduced memory performance when learning and retrieval contexts were incongruent, but not when they were congruent. Data represent means + standard errors of the means.

$\left..001, \eta^{2}=.80\right]$ over the four learning trials on Experimental Day 1 (see Table 2). Learning performance was comparable in men and women and in subjects in the stress and control, as well as the congruent and incongruent context, groups (all $F \mathrm{~s}<1)$. On average, the subjects scored 10.5 hits $(S E M=0.3)$ and needed about $48 \mathrm{sec}(S E M=2.0)$ for task completion on the last learning trial.

\section{Memory Performance}

Processing time. The effect of stress on the difference between the processing time on the test trial and the last learning trial depended on the context condition [treatment $\times$ context interaction, $F(1,64)=4.2, p=.045, \eta^{2}=$ $.06]$. As can be seen in Figure 3, the exposure to the SECPT increased this difference - that is, impaired memory performance - significantly in the incongruent context condition $[t(34)=2.9, p<.01, d=.96]$, but not in the congruent context condition [ $t(34)=0.2, p=.85, d=.07]$. We obtained a significant main effect for the context condition $\left[F(1,64)=4.7, p=.03, \eta^{2}=.07\right]$ and a trend for a treatment effect $\left[F(1,64)=3.1, p=.08, \eta^{2}=.05\right]$. Both were due primarily to the poor performance of stressed subjects in the incongruent context condition. Memory performance was unaffected by picture valence [neutral $(M \pm S E M$, in seconds), $5.5 \pm 1.5$; positive, $6.3 \pm 1.7$; negative, $7.3 \pm$ $\left.1.7 ; F(2,140)=0.25, p=.78, \eta^{2}<.01\right]$. There was no effect of sex, nor was there any further interaction between the factors (all $F \mathrm{~s}<2.2$, all $p \mathrm{~s}>.15$, all $\eta^{2} \mathrm{~s} \leq .03$ ).

Correct card locations. As is shown in Figure 4, the subjects who were stressed before retention testing remembered fewer card pairs than did controls when the learning and retrieval environments were incongruent $[t(34)=2.2$, $p<.05, d=.73$ ], whereas groups showed comparable memory performance when the learning and retrieval contexts were congruent $[t(34)=0.5, p=.65, d=.17$; treatment $\times$ context interaction, $F(1,64)=3.2, p=.078, \eta^{2}=$
.05]. Again, picture valence did not affect performance [neutral ( $M \pm S E M$, in seconds), $96.7 \pm 4.9$; positive, $100.8 \pm 7.4$; negative, $93.1 \pm 4.9 ; F(2,140)=0.35, p=$ $\left..71, \eta^{2}<.01\right]$. There were no main effects of stress, context, or sex, nor was there any further interaction between these factors (all $F_{\mathrm{s}}<1$, all $p \mathrm{~s}>.35$, all $\eta^{2} \mathrm{~s}<.02$ ).

\section{DISCUSSION}

Our primary goal in this experiment was to examine the effect of stress on the retrieval of context-dependent memories. We predicted that the detrimental influence of stress on memory retrieval would be attenuated if the learning and retrieval environments matched. The present findings confirmed this prediction. Stressed subjects performed worse than nonstressed controls when the learning and testing contexts were incongruent. This is in line with previous reports of impaired memory retrieval following stress (Buchanan et al., 2006; Kuhlmann et al., 2005). If, however, the learning and testing environments were matched, this detrimental effect of stress disappeared. Together with our previous findings (Schwabe, Bohringer, \& Wolf, 2009), these results provide strong evidence for an interaction of stress and context effects in memory processes (see Figure 5).

During learning, all available information-those related to the learning task itself, as well as those related to the learning environment - are integrated into the memory trace. This trace has to be reactivated at test, and it is reasonable to assume that the reactivation of the memory trace is easier the more learning-related information is present (Tulving \& Osler, 1968). The presentation of context cues leads to an enhanced activation of medial temporal lobe regions and prefrontal cortex areas that are relevant for memory integration (Mather et al., 2006; Rasch et al., 2007). Thus, the observed resistance of context-dependent memories to preretrieval stress may have been due to a stronger activation of medial temporal lobe regions, which may have overruled the increased retrieval threshold following stress (Joels et al., 2006). Alternatively, additional brain regions, such as the caudate nucleus, the amygdala, or sensory association cortices, may have supported the retrieval of context-dependent memories. Effects of the environmental context on memory are not necessarily explicit but can occur without explicit awareness of the context (Chun \& Jiang, 2003). Brain regions relevant for implicit memory, such as the caudate nucleus, contain significantly fewer stress hormone receptors than do medial temporal lobe areas (de Kloet et al., 1998), suggesting that they are less sensitive to the influence of stress and stress hormones. Indeed, previous evidence indicates that implicit memory tasks remain largely unaffected by stress (Kirschbaum et al., 1996; Lupien et al., 1997; Schwabe, Römer, et al., 2009). It is tempting to speculate that implicit memory systems were activated by the presentation of the learning context and, thus, contributed to the preserved retrieval performance after stress.

Although we focused mainly on the manipulation of the environmental (i.e., external) context, it is important to note that the presence or absence of stress can also be conceptualized as a change in internal context. According to this view, the subjects in the stress group were incongruent with 


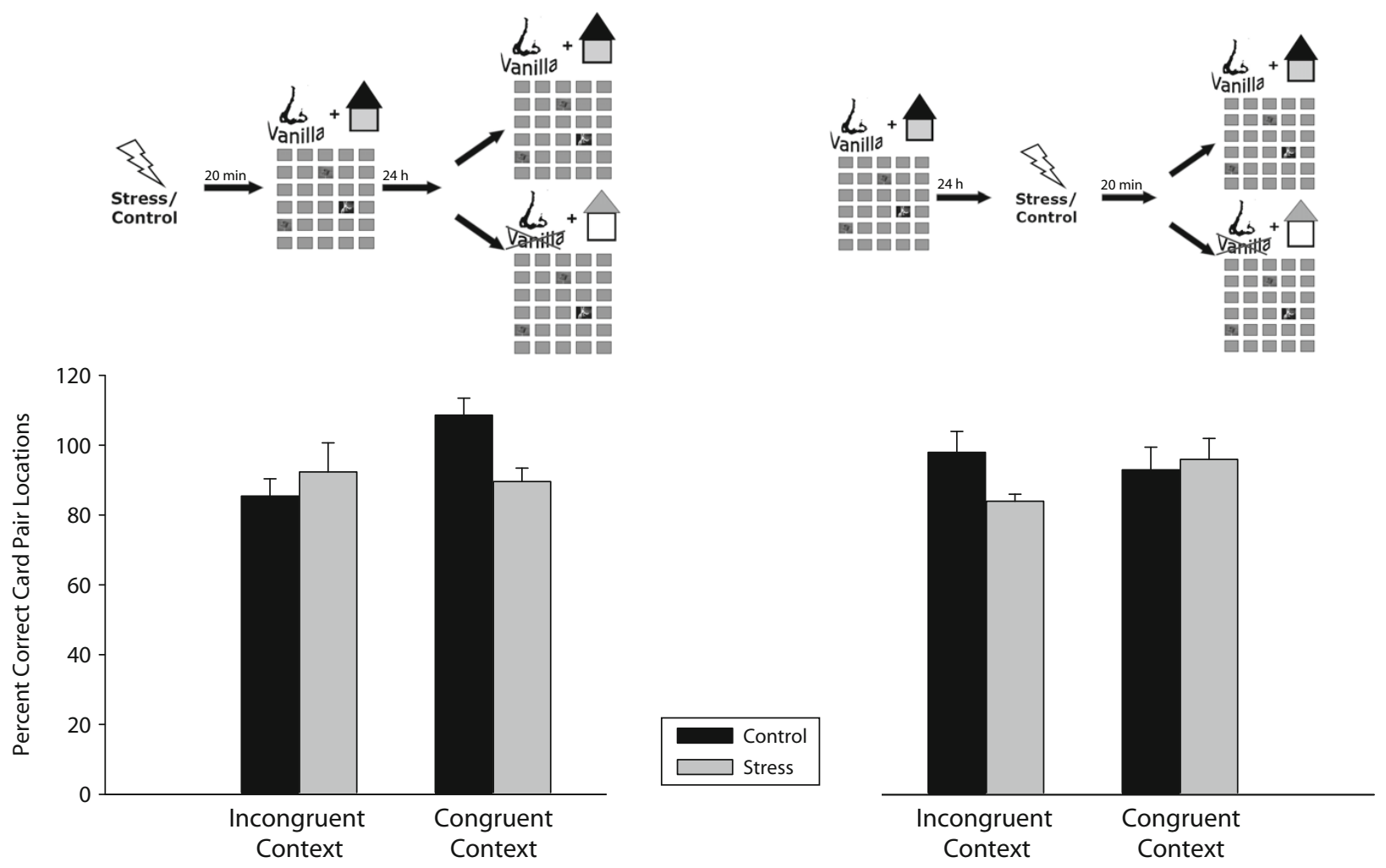

Figure 5. Comparison of the findings of our previous study (Schwabe, Bohringer, \& Wolf, 2009; left panel) and our present study (right panel). Stress before learning reduced the facilitative effect of congruent (odor and spatial) learning and testing contexts that was observed in the control group, which suggests that stress impairs the integration of contextual cues into the memory trace. Once a context-dependent memory is established, it can abolish the disruptive effect of preretrieval stress (observed in the incongruent context condition) if it is activated by the relevant context cues. The nose and the house represent the odor and the spatial environment, respectively, in the drawing. Note that recall values can be $>100 \%$ if more card pair locations are recalled in the retention test than on the last training trial. Data represent means + standard errors of the means.

respect to the internal context, since they were relaxed at encoding but stressed at testing, whereas the internal context was congruent in the control group. During the last decade, two phenomena have received a lot of attention that focuses on the impact of an individual's affective state or mood on memory: mood-congruent and mood-dependent memory (Eich, Kihlstrom, Bower, Forgas, \& Niedenthal, 2000). Mood-congruent memory refers to the observation that a given mood tends to facilitate the encoding and retrieval of material that shares the same emotional tone. Here, we did not find any differential effect of stress on the retrieval of neutral, positive, or negative items. Although this result might have been due, at least partly, to the relatively small number of items per emotion category, it suggests that mood congruency effects did not contribute significantly to the present findings. Mood-dependent memory refers to the observation that events encoded in a certain mood are subsequently most retrievable in that mood (Eich, 1995; Lewis \& Critchley, 2003). For example, Eich, Macaulay, and Ryan (1994) reported that subjects' memory for previously generated events was significantly better when the mood at testing, whether pleasant or unpleasant, matched the mood at event generation than when there was a mismatch. Thus, incongruent mood contexts may account for the detrimental impact of stress on memory retrieval, an explanation that has received surprisingly little attention in the research on stress effects on memory so far (but see Wolf, Schommer, Hellhammer, Reischies, \& Kirschbaum, 2002). Although we manipulated the subjects' affective state rather than their mood, the present results could suggest that the negative influence of an internal context mismatch may be compensated for by the presentation of incidentally processed, external context elements that cue memory. However, this interpretation is limited, since we have no overt measure of mood or state at the time of retrieval testing. Moreover, since retrieval testing took place about $20 \mathrm{~min}$ after the SECPT, it is uncertain whether the change in the internal state lasted until the beginning of memory testing.

Stress effects on memory are time dependent, with stress before retention testing having mostly impairing effects (Buchanan et al., 2006; de Quervain et al., 2000; Kuhlmann et al., 2005; Roozendaal, 2002). Our finding of reduced memory performance in stressed subjects who were tested in a context incongruent with the context at encoding supports this notion. However, there are also other studies indicating that stress before retention testing 
does not necessarily have detrimental effects but may even enhance memory retrieval (Domes, Heinrichs, Rimmele, Reichwald, \& Hautzinger, 2004; Luethi, Meier, \& Sandi, 2009; Schwabe, Römer, et al., 2009; Wolf et al., 2002). The present results suggest that this discrepancy in the literature might be solved by taking into account, next to the employed memory task and the time of testing (morning vs. evening; Lupien et al., 2002), the distinctiveness of the context at encoding and retrieval. Unfortunately, most authors do not explicitly mention whether learning and retention testing took place in the same environment, which makes it difficult to assess how much of the discrepancy in the literature on stress and memory retrieval can be explained by context effects.

Given that the congruency between learning and retrieval contexts abolished the stress-induced retrieval impairment, one might wonder why we found no overall environmental context effect - that is, why the context manipulation had no effect in the control group. One explanation for the absence of a general context effect takes into account the way in which memory was tested. Environmental context effects were most reliably shown when memory was assessed in free recall tests (Smith \& Vela, 2001). However, many studies did not show environmental context effects when recognition or cued recall tests were used - that is, tests that provide more noncontextual information than do free recall tests (e.g., Fernandez \& Glenberg, 1985; Jacoby, 1983; Smith, 1982). Interestingly, Godden and Baddeley (1980) also failed to replicate their previous findings with scuba divers (Godden \& Baddeley, 1975) when recognition tests were used instead of free recall tests. In the present study, memory performance was not assessed in a free recall test but, rather, in a cued-recall-like manner (the presentation of one card served as a cue for the corresponding second card), which could explain the lack of a context effect. Alternatively, the lack of a general context effect may well have been due to the excellent memory performance of the control group in the incongruent context condition, which did not leave much space for a further boost in performance. In future studies of stress effects on context-dependent memory, researchers might consider using free recall tests for memory assessment, which would most likely be more difficult than the cued-recall-like testing employed here and, thus, prevent performance at ceiling level.

We show that congruent contexts at learning and testing prevent the negative impact of stress on memory retrieval, yet we cannot show which of the different context elements contributed to this effect. The influence of the odor and the spatial environment cannot be disentangled in the present study. It is important to note that we did not aim to assess the contribution of the different context elements but to examine whether the reactivation of the distinct learning context - composed of an odor and a spatial environment - at test may attenuate stress effects on memory. Nevertheless, as has been nicely illustrated in Marcel Proust's Remembrance of Things Past and confirmed in scientific examinations, odor cues are particularly useful retrieval cues (Chu \& Downes, 2002; Herz, 1998). For instance, in one study visual, tactile, verbal, musical, and odor stimuli were used as memory cues. It was found that odor-evoked memories were significantly more emotional than those evoked by stimuli in the other modalities (Herz, 1998). Maybe odor cues are even potent enough to overcome the impact of stress on memory, independently of the spatial testing environment. Future studies could manipulate odor and spatial contexts independently of each other to dissect these influences.

In summary, the results of the present experiment indicate that the detrimental effects of stress on memory retrieval can be prevented when the environmental contexts at learning and testing match. These findings have important implications for educational concerns, such as whether the negative impact of test anxiety on exam scores might be reduced by testing students in the regular classroom, instead of in an unfamiliar room (whether learning bound to a certain context is desirable is another question). Furthermore, the present results may be of relevance to eyewitness testimony, which may be enhanced by returning the aroused eyewitness back to the scene of the witnessed event.

\section{AUTHOR NOTE}

This work was supported by DFG Grant SCHW 1357/2-1. We gratefully acknowledge the assistance of Anna Simon and Claudia Metzger during data collection. We thank Tobias Otto for the development of the computerized "Memory" game. Correspondence concerning this article should be addressed to L. Schwabe, Department of Cognitive Psychology, Ruhr University Bochum, Universitätsstrasse 150, 44780 Bochum, Germany (e-mail: lars.schwabe@rub.de).

\section{REFERENCES}

Buchanan, T. W., Tranel, D., \& Adolphs, R. (2006). Impaired memory retrieval correlates with individual differences in cortisol response but not autonomic response. Learning \& Memory, 13, 382-387.

CAhill, L., Gorski, L., \& Le, K. (2003). Enhanced human memory consolidation with post-learning stress: Interaction with the degree of arousal at encoding. Learning \& Memory, 10, 270-274.

Chu, S., \& Downes, J. J. (2002). Proust nose best: Odors are better cues of autobiographical memory. Memory \& Cognition, 30, 511-518.

Chun, M. M., \& JiAnG, Y. (2003). Implicit, long-term spatial contextual memory. Journal of Experimental Psychology: Learning, Memory, \& Cognition, 29, 224-234.

Chun, M. M., \& Phelps, E. A. (1999). Memory deficits for implicit contextual information in amnesic subjects with hippocampal damage. Nature Neuroscience, 2, 884-887.

Cohen, N. J., \& Eichenbaum, H. (1993). Memory, amnesia, and the hippocampal system. Cambridge, MA: MIT Press.

de Kloet, E. R., Vreugdenhil, E., Oitzl, M. S., \& Joels, M. (1998). Brain corticosteroid receptor balance in health and disease. Endocrine Reviews, 19, 269-301.

De Quervain, D. J., Aerni, A., \& Roozendaal, B. (2007). Preventive effect of $\beta$-adrenoceptor blockade on glucocorticoid-induced memory retrieval deficits. American Journal of Psychiatry, 164, 967-969.

de Quervain, D. J., Henke, K., Aerni, A., Treyer, V., McGaugh, J. L., Berthold, T., ET AL. (2003). Glucocorticoid-induced impairment of declarative memory retrieval is associated with reduced blood flow in the medial temporal lobe. European Journal of Neuroscience, 17, 1296-1302.

De Quervain, D. J., Roozendaal, B., Nitsch, R. M., McGaugh, J. L., \& Hock, C. (2000). Acute cortisone administration impairs retrieval of long-term declarative memory in humans. Nature Neuroscience, 3, 313-314

Diamond, D. M., Campbell, A. M., Park, C. R., Halonen, J., \& ZoLADZ, P. R. (2007). The temporal dynamics model of emotional memory 
processing: A synthesis on the neurobiological basis of stress-induced amnesia, flashbulb and traumatic memories, and the Yerkes-Dodson law. Neural Plasticity, 2007(Art. 60803). doi:10.1155/2007/60803

Dickerson, S. S., \& Kemeny, M. E. (2004). Acute stressors and cortisol responses: A theoretical integration and synthesis of laboratory research. Psychological Bulletin, 130, 355-391.

Domes, G., Heinrichs, M., Rimmele, U., Reichwald, U., \& HautZINGER, M. (2004). Acute stress impairs recognition for positive wordsAssociation with stress-induced cortisol secretion. Stress, 7, 173-181.

EIcH, E. (1995). Searching for mood dependent memory. Psychological Science, 6, 67-75.

Eich, E., Kinlstrom, J. F., Bower, G. H., Forgas, J. P., \& NiedenThal, P. M. (2000). Cognition and emotion. New York: Oxford University Press.

Eich, E., Macaulay, D., \& Ryan, L. (1994). Mood-dependent memory for events of the personal past. Journal of Experimental Psychology: General, 123, 201-215.

Fernandez, A., \& Glenberg, A. M. (1985). Changing environmental context does not reliably affect memory. Memory \& Cognition, 13, 333-345.

GLenberg, A. M. (1997). What memory is for. Behavioral \& Brain Sciences, 20, 1-55.

Godden, D. R., \& BAdDEley, A. D. (1975). Context-dependent memory in two natural environments: On land and underwater. British Journal of Psychology, 66, 325-331.

GoDDEN, D. R., \& BADDELEY, A. D. (1980). When does context influence recognition memory? British Journal of Psychology, 71, 99-104.

HeRZ, R. (1998). Are odors the best cues to memory? A cross-modal comparison of associative memory stimuli. In C. Murphy (Ed.), Olfaction and taste XII: An international symposium (Annals of the New York Academy of Sciences, Vol. 855, pp. 670-674). New York: New York Academy of Sciences.

JACOBY, L. L. (1983). Perceptual enhancement: Persistent effects of experience. Journal of Experimental Psychology: Learning, Memory, \& Cognition, 9, 21-38.

Joels, M., Pu, Z., Wiegert, O., Oitzl, M. S., \& Krugers, H. J. (2006). Learning under stress: How does it work? Trends in Cognitive Sciences, 10, 152-158.

Kirschbaum, C., \& Hellhammer, D. H. (1994). Salivary cortisol in psychoneuroendocrine research: Recent developments and applications. Psychoneuroendocrinology, 19, 313-333.

Kirschbaum, C., Kudielka, B. M., GaAb, J., Schommer, N. C., \& Hellhammer, D. H. (1999). Impact of gender, menstrual cycle phase, and oral contraceptives on the activity of the hypothalamus-pituaryadrenal axis. Psychosomatic Medicine, 61, 154-162.

Kirschbaum, C., Wolf, O. T., May, M., Wippich, W., \& Hellhammer, D. H. (1996). Stress- and treatment-induced elevations of cortisol levels associated with impaired declarative memory in healthy adults. Life Science, 58, 1475-1483.

Kuhlmann, S., Piel, M., \& Wolf, O. T. (2005). Impaired memory retrieval after psychosocial stress in healthy young men. Journal of Neuroscience, 25, 2977-2982.

Lang, P. J., Bradley, M. M., \& Cuthbert, B. N. (1997). International Affective Picture System (IAPS): Technical manual and affective ratings. Gainesville, FL: National Institute of Mental Health Center for the Study of Emotion and Attention.

Lewis, P. A., \& Critchley, H. D. (2003). Mood-dependent memory. Trends in Cognitive Sciences, 7, 431-433.

Luethi, M., Meier, B., \& SANDI, C. (2009). Stress effects on working memory, explicit memory, and implicit memory for neutral and emotional stimuli in healthy men. Frontiers in Behavioral Neuroscience, 2, 1-9.

Lupien, S. J., Gaudreau, S., Tchiteya, B. M., Maheu, F., Sharma, S., NAIR, N. P., ET AL. (1997). Stress-induced declarative memory impairment in healthy elderly subjects: Relationship to cortisol reactivity. Journal of Clinical Endocrinology \& Metabolism, 82, 2070-2075.

Lupien, S. J., \& LePaGe, M. (2001). Stress, memory, and the hippocampus: Can't live with it, can't live without it. Behavioural Brain Research, 127, 137-158.

Lupien, S. J., Wilkinson, C. W., Briere, S., Menard, C., Ng Ying KIN, N. M., \& NAIR, N. P. (2002). The modulatory effects of corticosteroids on cognition: Studies in young human populations. Psychoneuroendocrinology, 27, 401-416.
Mather, M., Mitchell, K. J., Raye, C. L., Novak, D. L., Greene, E. J., \& Johnson, M. K. (2006). Emotional arousal can impair feature binding in working memory. Journal of Cognitive Neuroscience, 18, 614-625.

McGaugh, J. L., \& RoozendaAl, B. (2002). Role of adrenal stress hormones in forming lasting memories in the brain. Current Opinion in Neurobiology, 12, 205-210.

Oei, N. Y. L., Elzinga, B. M., Wolf, O. T., De Ruiter, M. B., DamoiSEAUX, J. S., KuiJer, J. P. A., ET AL. (2007). Glucocorticoids decrease hippocampal and prefrontal activation during declarative memory retrieval in young men. Brain Imaging \& Behavior, 1, 31-41.

Payne, J. D., Jackson, E. D., Ryan, L., Hoscheidt, S., Jacobs, W. J., \& NADEL, L. (2006). The impact of stress on neutral and emotional aspects of episodic memory. Memory, 14, 1-16.

Rasch, B., Büchel, C., GaIs, S., \& Born, J. (2007). Odor cues during slow wave sleep prompt declarative memory consolidation. Science, 315, 1426-1429.

RoozendaAL, B. (2002). Stress and memory: Opposing effects of glucocorticoids on memory consolidation and memory retrieval. Neurobiology of Learning \& Memory, 78, 578-595.

RoozendaAl, B., De Quervain, D. J., Schelling, G., \& McGaugh, J. L. (2004). A systemically administered beta-adrenoceptor antagonist blocks corticosterone-induced impairment of contextual memory retrieval in rats. Neurobiology of Learning \& Memory, 81, 150-154.

Roozendaal, B., OKuda, S., Van der Zee, E. A., \& McGaugh, J. L. (2006). Glucocorticoid enhancement of memory requires arousalinduced noradrenergic activation in the basolateral amygdala. Proceedings of the National Academy of Sciences, 103, 6741-6746.

Rose, J., Otтo, T., \& DitTrich, L. (2008). The Biopsychology-Toolbox: A free, open-source Matlab-toolbox for the control of behavioral experiments. Journal of Neuroscience Methods, 175, 104-107.

Schwabe, L., Bohringer, A., Chatterjee, M., \& Schachinger, H. (2008). Effects of pre-learning stress on memory for neutral, positive and negative words: Different roles of cortisol and autonomic arousal. Neurobiology of Learning \& Memory, 90, 44-53.

Schwabe, L., Bohringer, A., \& Wolf, O. T. (2009). Stress disrupts context-dependent memory. Learning \& Memory, 16, 110-113.

Schwabe, L., Haddad, L., \& Schachinger, H. (2008). HPA axis activation by a socially evaluated cold pressor test. Psychoneuroendocrinology, 33, 890-895.

Schwabe, L., Römer, S., Richter, S., Dockendorf, S., Bilak, B., $\&$ SCHACHINGeR, H. (2009). Stress effects on declarative memory retrieval are blocked by a $\beta$-adrenoceptor antagonist in humans. Psychoneuroendocrinology, 34, 446-454.

Smeets, T., Otgaar, H., Candel, I., \& Wolf, O. T. (2008). True or false? Memory is differentially affected by stress-induced cortisol elevations and sympathetic activity at consolidation and retrieval. Psychoneuroendocrinology, 33, 1378-1386.

Sмiтн, S. M. (1982). Enhancement of recall using multiple environmental contexts during learning. Memory \& Cognition, 10, 405-412.

Smith, S. M., \& Vela, E. (2001). Environmental context-dependent memory: A review and meta-analysis. Psychonomic Bulletin \& Review, 8, 203-220.

Tulving, E., \& Osler, S. (1968). Effectiveness of retrieval cues in memory for words. Journal of Experimental Psychology, 77, 593-601.

Tulving, E., \& Thompson, D. M. (1973). Encoding specificity and retrieval processes in episodic memory. Psychological Review, 80, 352-373.

Wagner, A. D., Desmond, J. E., Glover, G. H., \& Gabrieli, J. D. E. (1998). Prefrontal cortex and recognition memory: Functional-MRI evidence for context-dependent retrieval processes. Brain, 121, 1985-2002.

WoLF, O. T. (2008). The influence of stress hormones on emotional memory: Relevance for psychopathology. Acta Psychologica, 127, 513-531.

Wolf, O. T., Schommer, N. C., Hellhammer, D. H., Reischies, F. M., $\&$ Kirschbaum, C. (2002). Moderate psychosocial stress appears not to impair recall of words learned 4 weeks prior to stress exposure. Stress, 5, 59-64.

(Manuscript received May 4, 2009; revision accepted for publication June 15, 2009.) 